\title{
Characterization of pulmonary protein profiles in response to zinc oxide nanoparticles in mice: a 24-hour and 28-day follow-up study
}

This article was published in the following Dove Press journal:

International Journal of Nanomedicine

27 July 2015

Number of times this article has been viewed

Chih-Hong Pan, 1,2,* Kai-Jen Chuang, ${ }^{3,4, *}$ Jen-Kun Chen, ${ }^{5}$ Ta-Chih Hsiao, ${ }^{6}$ Ching-Huang Lai, ${ }^{2}$ Tim P Jones, ${ }^{7}$ Kelly A BéruBé, ${ }^{8}$ Gui-Bing Hong, ${ }^{9}$ Kin-Fai Ho, ${ }^{10,11}$ Hsiao-Chi Chuang ${ }^{12,13}$

'Institute of Occupational Safety and Health, Council of Labor Affairs, Executive Yuan, ${ }^{2}$ School of Public Health, National Defense Medical Center, ${ }^{3}$ School of Public Health, College of Public Health and Nutrition, ${ }^{4}$ Department of Public Health, School of Medicine, College of Medicine, Taipei Medical University, Taipei, ${ }^{5}$ Institute of Biomedical Engineering and Nanomedicine, National Health Research Institutes, Miaoli, ${ }^{6} \mathrm{Graduate}$ Institute of Environmental Engineering, National Central University, Taoyuan, Taiwan; ${ }^{7} \mathrm{School}$ of Earth and Ocean Sciences, ${ }^{8}$ School of Biosciences, Cardiff University, Cardiff, Wales, UK; ${ }^{9}$ Department of Chemical Engineering and Biotechnology, National Taipei University of Technology, Taipei, Taiwan; ${ }^{10}$ Jockey Club School of Public Health and Primary Care, The Chinese University of Hong Kong, Hong Kong, People's Republic of China; "Shenzhen Municipal Key Laboratory for Health Risk Analysis, Shenzhen Research Institute, The Chinese University of Hong Kong, Shenzhen, People's Republic of China ${ }^{12}$ School of Respiratory Therapy, College of Medicine, ${ }^{13}$ Division of Pulmonary Medicine. Department of Internal Medicine, Shuang Ho Hospital, Taipei Medical University, Taipei, Taiwan

*These authors contributed equally to this work

Correspondence: Hsiao-Chi Chuang Taiwan Cardio Pulmonary Research (T-CPR) Group, School of Respiratory Therapy, College of Medicine, Taipei Medical University, 250 Wuxing Street, Taipei City II0, Taiwan Tel +88622736 I66I ext 3515

Fax +886 22739 I| 43

Email r92841005@ntu.edu.tw
Abstract: Although zinc oxide nanoparticles (ZnONPs) are recognized to cause systemic disorders, little is known about the mechanisms that underlie the time-dependent differences that occur after exposure. The objective of this study was to investigate the mechanistic differences at 24 hours and 28 days after the exposure of BALB/c mice to ZnONPs via intratracheal instillation. An isobaric tag for the relative and absolute quantitation coupled with liquid chromatography/tandem mass spectrometry was used to identify the differential protein expression, biological processes, molecular functions, and pathways. A total of 18 and 14 proteins displayed significant changes in the lung tissues at 24 hours and 28 days after exposure, respectively, with the most striking changes being observed for S100-A9 protein. Metabolic processes and catalytic activity were the main biological processes and molecular functions, respectively, in the responses at the 24-hour and 28-day follow-up times. The glycolysis/gluconeogenesis pathway was continuously downregulated from 24 hours to 28 days, whereas detoxification pathways were activated at the 28-day time-point after exposure. A comprehensive understanding of the potential time-dependent effects of exposure to ZnONPs was provided, which highlights the metabolic mechanisms that may be important in the responses to $\mathrm{ZnONP}$.

Keywords: glycolysis, iTRAQ, metabolism, nanoparticles, S100-A9

\section{Introduction}

Nanoparticles (NPs), defined as particles less than $100 \mathrm{~nm}$ in diameter, are commonly used in commercial products and biomedicine; however, the potential bioreactivity and mechanisms for processes of concern with respect to safety have not been well characterized. Zinc oxide NPs (ZnONPs), for example, may be found in many commercial nanomaterials and in ambient air due to combustion sources. ${ }^{1}$ The generation of high concentrations of $\mathrm{ZnONPs}$ has been reported in manufacturing processes, including in brass founding and welding or cutting galvanized sheet metal. ${ }^{2}$ The actual exposure to metal fumes depends on the conditions in the occupational setting, but the permissible exposure limit for occupational exposure to $\mathrm{ZnO}$ in the United States is set at $5 \mathrm{mg} / \mathrm{m}^{3}$ respirable dust. Metal fume fever is an occupational disease that is caused by the inhalation of $\mathrm{ZnO}{ }^{3}$ This illness leads to respiratory and systemic syndromes that often occur in workers exposed to zinc metal fumes. ${ }^{1,4}$ Clinical observations have indicated that metal fume fever is initiated a few hours after $\mathrm{ZnO}$ exposure, with illness following within 24 hours. Acquired tolerance occurs after repeated exposures, but the mechanisms remain unclear, particularly for $\mathrm{ZnONPs.}$

High-throughput proteomic and genomic approaches can provide an unbiased assessment of the biological responses to stressors. ${ }^{5}$ Proteomic techniques, for example, are powerful tools with which to determine differential protein expression. Traditionally, 
this technique relies on high-resolution two-dimensional electrophoresis to separate the proteins. This process has limitations with respect to the determination of 1) highly acidic or basic proteins, 2) proteins of low and high abundance, and 3) hydrophobic proteins. ${ }^{6}$ These limitations can be overcome using liquid chromatography-tandem mass spectrometry (LC-MS/ MS), which is a highly sensitive method to rapidly and automatically identify the proteins present in mixtures. ${ }^{6}$ Isobaric tags for relative and absolute quantitation (iTRAQ) is an isobaric labeling method that is used to quantitatively assess protein expression. This method increases throughput as well as reducing experimental error. ${ }^{7}$ The application of LC-MS/MS and iTRAQ approaches to toxicological investigations may offer comprehensive information for environmental and occupation health. For example, intracellular signaling molecules involved in calcium regulation in response to hydroxyapatite NPs were identified using the iTRAQ-coupled LC-MS/MS approach. ${ }^{8}$

The symptoms of $\mathrm{ZnO}$ metal fume fever often occur within 24 hours, and tolerance is acquired after repeated exposures. ${ }^{1}$ In a rat model, pulmonary impairment and degeneration as well as necrosis of the myocardium were detected after 30 days of repeated exposure to ZnONPs. ${ }^{9}$ Time-dependent responses, including mitochondrial dysfunction and oxidative stress, were observed in rat alveolar epithelial cell monolayers. ${ }^{10}$ Together, these results imply that the health effects of ZnONP exposure may depend on the time following exposure. Therefore, the objective of this study was to investigate the time-dependent mechanistic differences at acute (24 hours) and subacute (28 days after single $\mathrm{ZnONP}$ exposure) time intervals in mice. The iTRAQcoupled LC-MS/MS approach was used to identify the differential protein expression, biological processes, molecular functions, and pathways.

\section{Materials and methods $\mathrm{ZnONP}$ and reagent sources}

The ZnONP was obtained from the Nanostructured and Amorphous Materials, Inc. (Houston, TX, USA). All of the other reagents were obtained from Sigma (MO, USA), if the source is not explicitly stated.

\section{Field emission-scanning electron microscopy and energy-dispersive $\mathrm{X}$-ray microanalysis}

An Inspect ${ }^{\mathrm{TM}}$ field emission-scanning electron microscope (FESEM; JEOL 2100, Jeol, Japan) and an energy-dispersive $\mathrm{X}$-ray (EDX) microanalysis instrument were used to characterize the ZnONPs, according to our previous report. ${ }^{9}$ Briefly, the ZnONPs were investigated via adhesion to $12 \mathrm{~mm}$ carbon adhesive tabs on $13 \mathrm{~mm}$ aluminum SEM stubs. The SEM stubs were coated with platinum $(\mathrm{Pt})$, with an average thickness of $10 \mathrm{~nm}$ using a sputter coater. FESEM images were obtained using the following conditions: an accelerating voltage of $15 \mathrm{kV}$ and a 2.5 spot size. Elemental analysis was conducted using the EDX Genesis Microanalysis System (EDAX Inc., Mahwah, NJ, USA).

\section{Animals}

Six-week-old female BALB/c mice were obtained from BiOLASCO (Taipei, Taiwan). The mice were maintained at $22^{\circ} \mathrm{C} \pm 2{ }^{\circ} \mathrm{C}$ and $55 \% \pm 10 \%$ relative humidity in a light/dark cycle (12/12 hours). The animals were housed in plastic cages and were provided the Lab Diet 5001 (PMI Nutrition International, Shoreview, MN, USA) and water ad libitum during acclimatization, preexposure, and postexposure. Approval was obtained and the animal experiments were performed in compliance with the animal and ethics review committee of the Laboratory Animal Centre at the Taipei Medical University (Taiwan).

\section{Experimental design}

The experimental design is shown in Figure 1. To investigate the acute and subacute effects of single pulmonary exposure of ZnONPs, 24-hour follow-up and 28-day follow-up experiments were performed after exposure. The mice were randomly divided into two groups (control $[\mathrm{n}=6]$ and exposure $[n=6]$ ) for each experiment. In the 24-hour follow-up group, the mice were administered $100 \mu \mathrm{g} / \mathrm{lung}$ (mouse) ZnONPs $(50 \mu \mathrm{L})$ in phosphate-buffered saline (PBS) with 5\% bovine serum albumin (BSA), whereas ZnONP-free PBS with 5\% BSA was used as vehicle control (Figure 1A). In the 28-day follow-up group, the mice were administered $100 \mu \mathrm{g} /$ lung (mouse) ZnONPs $(50 \mu \mathrm{L})$ in PBS with 5\% BSA, whereas ZnONP-free PBS with 5\% BSA was used as vehicle control (Figure 1B). Intratracheal (IT) instillation of the samples was performed under light anesthesia induced by $2 \%$ isoflurane vapor $(2 \mathrm{cc} /$ min), using a rodent anesthesia machine (Northern Vaporiser; Skipton, England, UK). The mice in the 24-hour follow-up group were euthanized on day 1 (24 hours after exposure; acute), whereas the mice in the 28-day follow-up group were euthanized on day 28 (28 days after exposure; subacute).

\section{Lung tissue collection}

The animal necropsies were performed as described previously. ${ }^{11}$ The lung tissues were excised after euthanasia with a single intraperitoneal injection of sodium pentobarbital $(200 \mathrm{mg} / \mathrm{mL})$. For protein isolation, the lung tissue was homogenized in radioimmunoprecipitation assay (RIPA) 


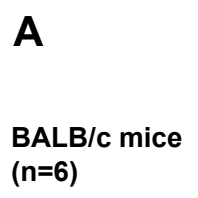

Group 1

Group 2

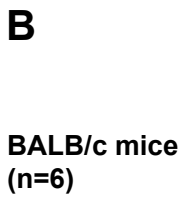

Group 1

Group 2

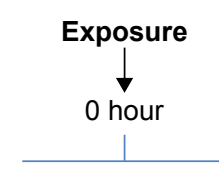

PBS

$100 \mu \mathrm{g} / \mathrm{lung}$ ZnONP

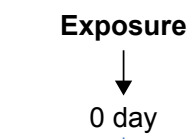

PBS

$100 \mu \mathrm{g} / \mathrm{lung} \mathrm{ZnONP}$

Figure I Overview of the two experimental designs for analysis of the effects of $\mathrm{ZnONP}$ exposure in mice.

Notes: (A) A dose of $100 \mu \mathrm{g} /$ lung $\mathrm{ZnONPs}$ was administered via IT instillation ( $n=6$ ); PBS was used as vehicle control ( $n=6)$. The animals were euthanized 24 hours after exposure. (B) A dose of $100 \mu \mathrm{g} /$ lung $\mathrm{ZnONPs}$ was administered via IT instillation $(n=6)$; PBS was used as vehicle control $(n=6)$. The animals were euthanized 28 days after exposure.

Abbreviations: ZnONPs, zinc oxide nanoparticles; IT, intratracheal; PBS, phosphate-buffered saline.

buffer (Sigma) with Complete ${ }^{\mathrm{TM}}$ protease inhibitor (Roche Diagnostics, Basel, Switzerland), according to the manufacturers' instructions.

\section{Protein digestion and iTRAQ labeling}

Sample pooling is a strategy commonly used to minimize individual variation in proteomic studies. ${ }^{12}$ The proteins isolated from the lungs within the same group $(\mathrm{n}=6)$ were pooled: 24-hour control versus 24-hour ZnONP and 28-day control versus 28-day ZnONP. Albumin and IgG were depleted using a Qproteome Albumin/IgG Depletion Kit (Qiagen, Germantown, MD, USA). One hundred micrograms of each sample was precipitated with acetone at $-20^{\circ} \mathrm{C}$ for 2 hours and then redissolved in $0.5 \mathrm{M}$ triethylammonium bicarbonate (TEAB) as a cleanup process. Subsequent reduction, alkylation, digestion, and iTRAQ labeling (Applied Biosystems, Grand Island, NY, USA) were performed according to the manufacturer's protocol. Briefly, the protein samples were reduced in $5 \mathrm{mM}$ tris-(2-carboxyethyl)phosphine (TCEP) at $60^{\circ} \mathrm{C}$ for 1 hour, and the cysteine groups were blocked by incubation with a $10 \mathrm{mM}$ methyl methanethiosulfonate (MMTS) solution at room temperature for 10 minutes. The samples were digested with $10 \mu \mathrm{g}$ trypsin at $37^{\circ} \mathrm{C}$ for 16 hours and then labeled with the iTRAQ tag (114 for control and 115 for $\mathrm{ZnONP}$ exposure). The samples were combined, dried using a speedvac, redissolved in $50 \mu \mathrm{L}$ of $5 \%$ acetone in $0.1 \%$ formic acid, and subjected to further analysis.

\section{LC-MS/MS}

The iTRAQ-labeled samples were combined and analyzed using a nano LC-Quadruple Orbitrap equipped with high-energy collision dissociation (HCD) technology. ${ }^{13}$ Briefly, the samples were analyzed using a $Q$ Exactive mass spectrometer (Thermo Fisher Scientific, Bremen, Germany) coupled with an UltiMate 3000 RSLC system (Dionex, Sunnyvale, CA, USA). The HCD fragmentation mode was used to generate MS and MS/MS spectra. The LC separation was performed using $\mathrm{C} 18$ columns $(75 \mu \mathrm{m} \times 150 \mathrm{~mm}, 2 \mu \mathrm{m}$, $100 \AA$; Acclaim PepMap RSLC, Dionex) using the following conditions for the full MS scans: $m / z$ 380-2,000, 380-600, $600-800,800-1,200$, and 1,200-2,000. The ten most intense ions from the MS scans were subjected to fragmentation for the MS/MS spectra. The raw data were processed into peak lists by searching the Proteome Discoverer 1.4 for Mascot database using search parameters including variable modifications for deamidation (NQ) and oxidation (M) and fixed modifications for methylthio (C), iTRAQ 4plex (N-terminal), and iTRAQ 4plex (K). The maximum mass tolerance was set to $10 \mathrm{ppm}$ for the precursor ions and to $0.05 \mathrm{Da}$ for the fragment ions. Only peptides with Mascot scores above 30 at the $95 \%$ confidence level were further considered. The criteria used for selection of differentially expressed proteins included the following: at least two unique high-scoring peptides in the protein; $P<0.05$ across two independent iTRAQ experiments; and a protein ratio of $>1.3$ or $<0.77 .^{14}$

\section{Protein functional analyses}

The upregulated and downregulated proteins in the 24-hour and 28-day groups were subjected to functional pathway analyses using the Protein ANalysis THrough Evolutionary Relationships (PANTHER) classification system 
(http://www.pantherdb.org/) to determine the biological processes and molecular functions of the identified proteins. ${ }^{15}$ The Database for Annotation, Visualization and Integrated Discovery (DAVID) gene functional analysis tool (http:// david.abcc.ncifcrf.gov/) was used to determine the pathways involved in the 24-hour and 28-day groups. ${ }^{16,17}$ For the DAVID analysis, an enhanced score $\geq 1.3$, set as the threshold, was considered to be significant, as described in a previous report. ${ }^{18}$

\section{Protein network analysis}

The differentially expressed proteins were subjected to protein network analysis using the NetworkAnalyst (http:// www.networkanalyst.ca/NetworkAnalyst/) to investigate the protein network of the identified proteins. ${ }^{19}$ The UniProt accession database was used to access the 18 and 14 proteins which showed significant changes in the lung tissues at 24 hours and 28 days after exposure, respectively. These proteins were uploaded and mapped against the Mus musculus reference dataset to extract and summarize the protein network associated with individual genes/proteins or groups of genes/proteins.

\section{Results \\ Characterization of ZnONPs}

$\mathrm{ZnONPs}$ with an average diameter of $20 \mathrm{~nm}$ were used in this study. The basic characteristics of the $\mathrm{ZnONP}$ as provided by the supplier were $99.5 \mathrm{wt} \%$ purity and $50 \mathrm{~m}^{2} / \mathrm{g}$ specific surface area. The shapes of most of the ZnONPs were hexagonal columns of various lengths (Figure 2). Only $\mathrm{Zn}$ and $\mathrm{O}$ were detected in the $\mathrm{ZnONP}$, except for $\mathrm{C}$ (from the carbon tab) and Pt (from the coating material) (Figure 2).

\section{iTRAQ-labeling protein expression}

A Total of 304 and 246 proteins were identified for the 24-hour and 28-day studies, respectively, among which 280 and 141 proteins were quantified for iTRAQ-labeled samples, respectively. These proteins are listed in the Supplementary materials as Tables S1 (http://www.dovepress.com/get supplementary file.php?f=82979.pdf) and S2 (http://www.dovepress.com/ get supplementary file.php? $\mathrm{f}=82979$ 1.pdf). A total of 18 and 14 proteins displayed significant changes in the lung tissues at 24 hours and 28 days after exposure, respectively. The differentially expressed proteins were identified as follows: the
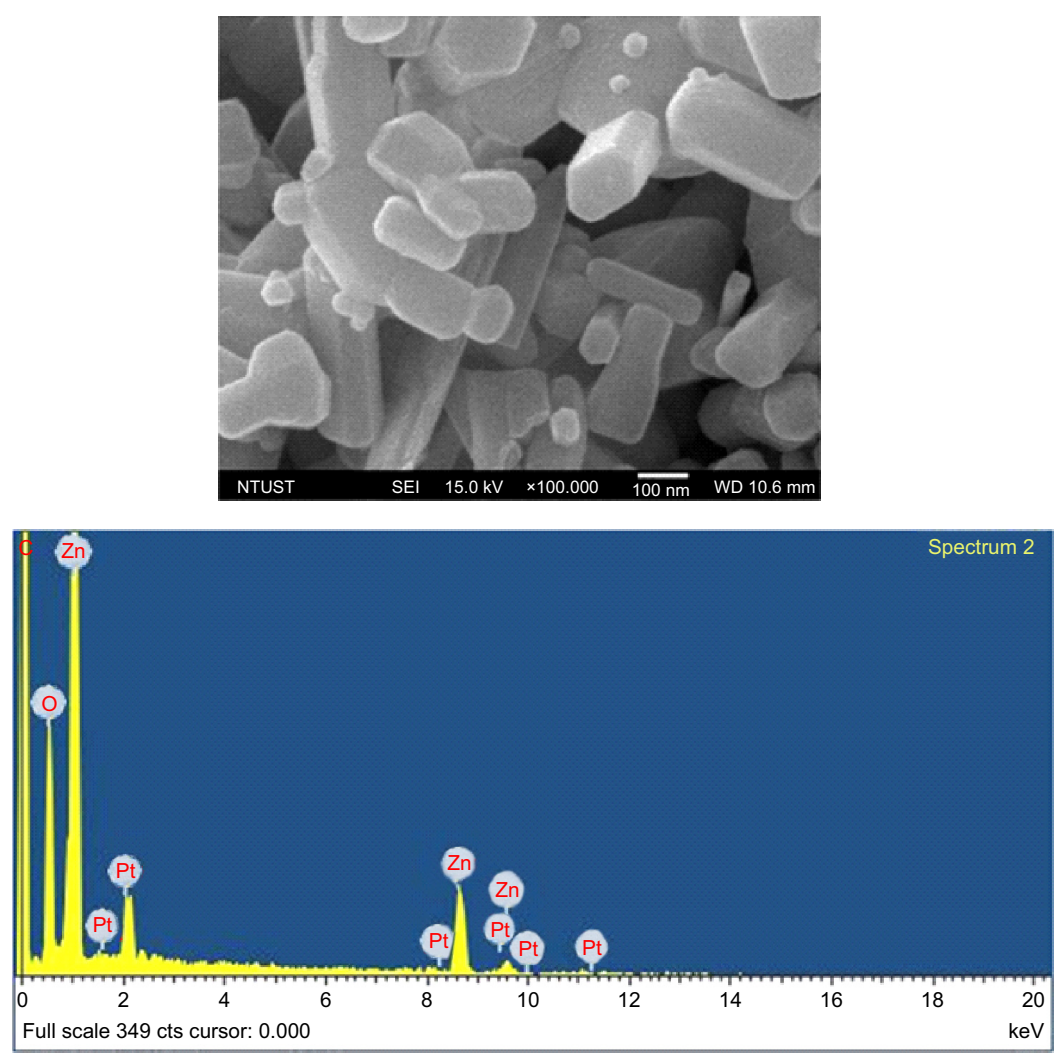

Figure 2 SEM photographs of commercial 20 nm-diameter ZnONPs.

Notes: The shapes of the ZnONPs were hexagonal columns of various lengths. Only $\mathrm{Zn}$ and $\mathrm{O}$ detected in the $\mathrm{ZnONP}$ with the addition of $\mathrm{C}$ (from the carbon stick) and Pt (from the coating material) as indicated by EDX analysis.

Abbreviations: SEM, scanning electron microscopy; ZnONPs, zinc oxide nanoparticles; EDX, energy-dispersive X-ray. 
downregulated proteins in the 24-hour group were aconitate hydratase mitochondrial (ratio $=0.573$ ), ATP-citrate synthase (ratio $=0.699$ ), calpastatin (ratio $=0.762$ ), carbonic anhydrase 3 (ratio $=0.554)$, citrate synthase $($ ratio $=0.634)$, creatine kinase B-type (ratio $=0.703)$, cytochrome c somatic $($ ratio $=0.729)$, electron transfer flavoprotein subunit $\alpha$ (ratio $=0.694$ ), fatty acid synthase (ratio $=0.654$ ), fatty acid-binding protein (ratio $=0.287$ ), long-chain specific acyl-CoA dehydrogenase (ratio $=0.715)$, malate dehydrogenase $($ ratio $=0.737)$, myoglobin (ratio $=0.755$ ), and uteroglobin (ratio $=0.747$ ), while the upregulated proteins included annexin A1 (ratio=1.668), apolipoprotein A-II (ratio =1.364), cysteine-rich protein 2 $($ ratio $=1.530)$, and protein S100-A9 $($ ratio $=2.090)($ Figure 3$)$. The downregulated proteins in the 28-day group included $\beta$-enolase $($ ratio $=0.594)$, calmodulin $($ ratio $=0.673)$, carbonic anhydrase 3 (ratio $=0.375$ ), creatine kinase M-type (ratio $=0.578)$, cytochrome c somatic $($ ratio $=0.708)$, myoglobin (ratio $=0.717$ ), and vimentin (ratio $=0.741$ ), and the upregulated proteins included actin cytoplasmic 1 $($ ratio $=1.425), \beta$-actin-like protein 2 (ratio $=1.462)$, chloride intracellular channel protein 5 (ratio $=1.420)$, cysteine-rich protein 2 (ratio $=1.497)$, hemoglobin subunit $\beta-1$ (ratio $=1.400)$, hemoglobin subunit $\beta-2$ (ratio =1.389), and tubulin $\alpha-1 \mathrm{~A}$ chain (ratio $=1.531$ ) (Figure 3 ). Fatty acid-binding protein and protein S100-A9 were the most down- and upregulated proteins in response to $\mathrm{ZnONP} 24$ hours after exposure, respectively. Carbonic anhydrase 3 and tubulin $\alpha-1 \mathrm{~A}$ chain were the most down- and upregulated proteins in the 28-day group, respectively. In comparing the 24-hour and 28-day follow-up groups, we observed that the expression of carbonic anhydrase 3 , cytochrome c somatic, myoglobin, and vimentin was downregulated in both groups, whereas expression of cysteine-rich protein 2 was upregulated in both groups.

\section{Biological processes and molecular functions}

All the downregulated and upregulated proteins among the 280 and 141 proteins in the 24-hour and 28-day groups, respectively, were analyzed to evaluate their implications for biological processes and molecular functions. Apoptosis, biological adhesion, biological regulation, cellular component organization or biogenesis, cellular process, developmental process, immune system process, localization, metabolic process, multicellular organismic process, reproduction, and response to stimuli were the main biological processes indicated by the down- and upregulation responses at the 24-hour and

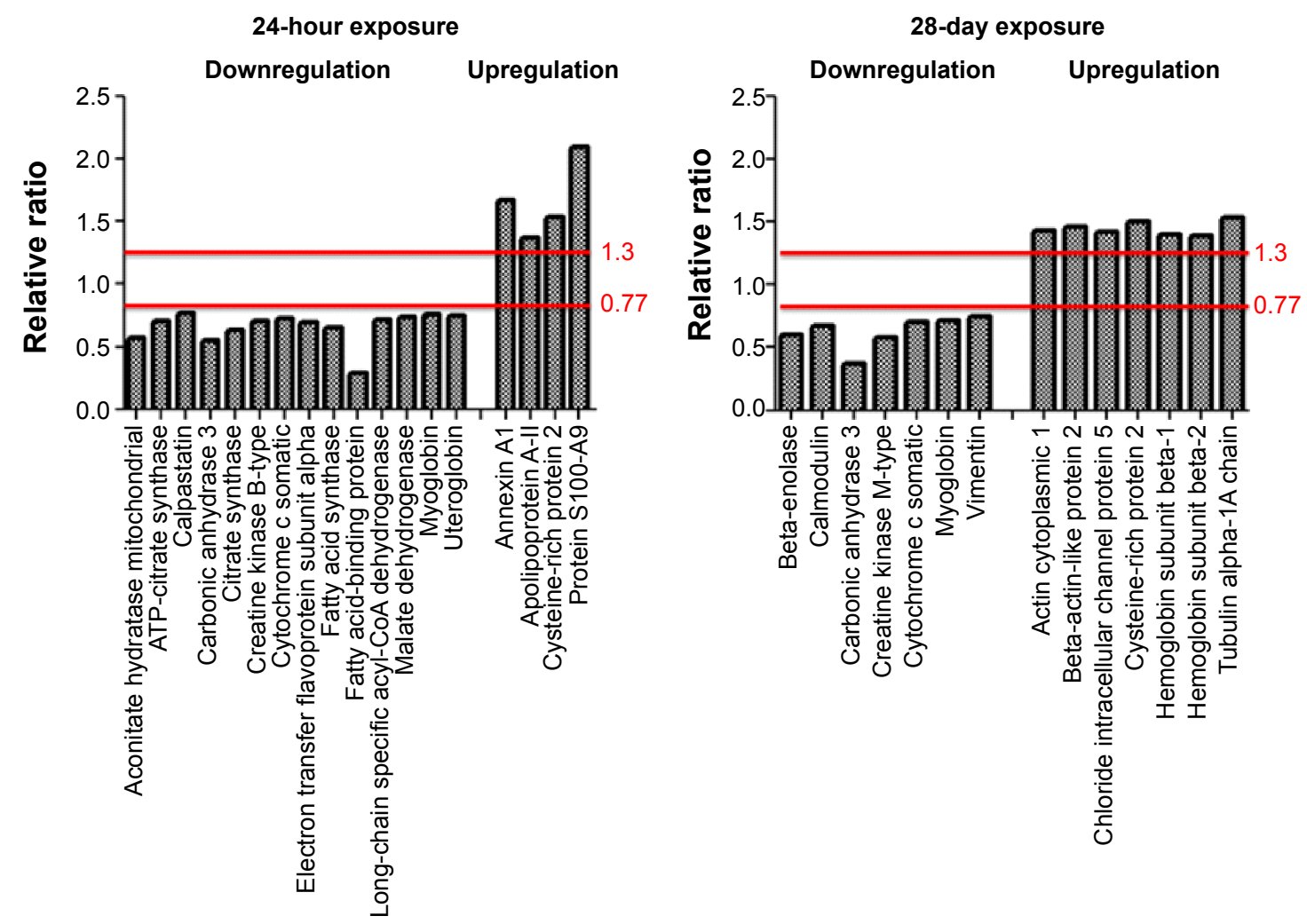

Figure 3 Expression of protein down- and up-regulation in lung tissue after exposure of $\mathrm{ZnONP}$.

Notes: The iTRAQ-coupled LC-MS/MS approach identified a total of 18 and 14 proteins in the lung tissues in the 24-hour follow-up and 28-day follow-up groups, respectively, that displayed significant down- or upregulation after exposure to the ZnONPs. A protein ratio of $>1.3$ or $<0.77$ was considered to be a significant change. ${ }^{14}$ Abbreviations: ZnONPs, zinc oxide nanoparticles; LC-MS/MS, liquid chromatography-tandem mass spectrometry; iTRAQ, isobaric tags for relative and absolute quantitation. 

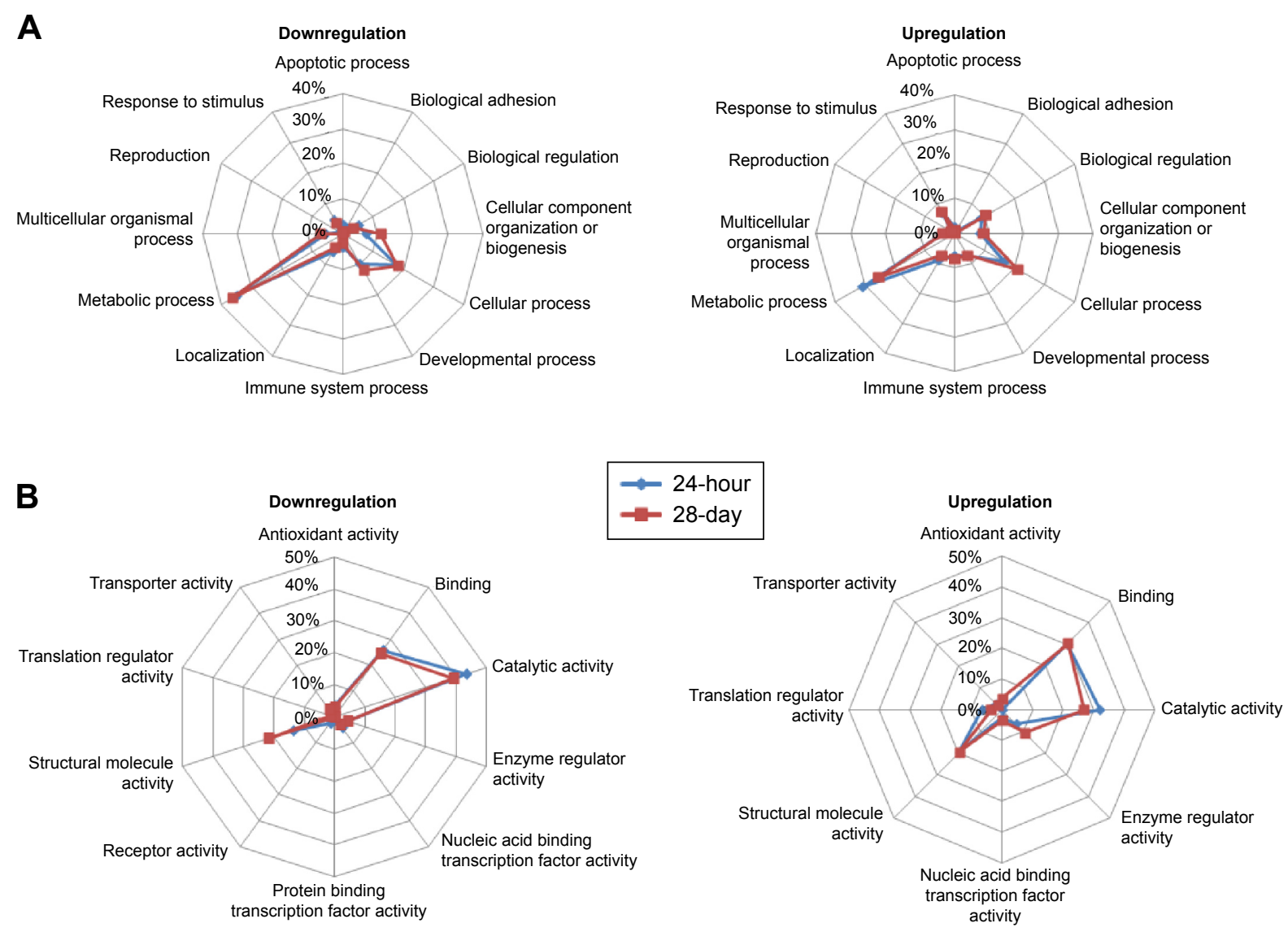

Figure 4 Biological processes and molecular functions associated with downregulation and upregulation of proteins after 24 hours and 28 days exposure to $\mathrm{ZnONP}$. Notes: (A) Biological processes associated with the downregulation and upregulation of proteins in the 24-hour and 28-day responses after exposure to ZnONP. (B) Molecular functions associated with the downregulation and upregulation of proteins in the 24-hour and 28-day responses after exposure to ZnONP. The biological processes and molecular functions were determined using PANTHER analysis.

Abbreviations: ZnONP, zinc oxide nanoparticle; PANTHER, Protein ANalysis THrough Evolutionary Relationships.

28-day follow-up times after ZnONP exposure (Figure 4A). Metabolic processes (35\%-36\% downregulated and $25 \%-31 \%$ upregulated) and cellular processes $(17 \%-18 \%$ downregulated and $16 \%-21 \%$ upregulated) were the most two important biological responses represented by the down- and upregulated proteins in the 24-hour and 28-day groups. Antioxidant activity, binding, catalytic activity, enzyme regulator activity, nucleic acid-binding transcription factor activity, structural molecule activity, translation regulator activity, and transporter activity were observed in both the downregulated and upregulated classes of proteins in both the 24-hour and 28-day groups (Figure 4B). Protein-binding transcription factor activity and receptor activity were found among the downregulated proteins, but not in upregulated proteins. Binding (25\%-26\% downregulated and 30\% upregulated), catalytic activity (39\%-43\% downregulated and $27 \%-32 \%$ upregulated), and structural molecule activity (14\%-22\% downregulated and $20 \%$ upregulated) were the important molecular functions altered in the 24-hour and 28-day responses to $\mathrm{ZnONP}$ exposure.

\section{Pathways for 24 hours and 28 days follow-up of exposure}

Downregulated proteins involved in 24 pathways were identified in the 24-hour group, and 18 of these pathways were considered to be significant (Figure 5). These included glycolysis/gluconeogenesis, citrate cycle (TCA cycle), glutathione metabolism, pyruvate metabolism, metabolism of xenobiotics by cytochrome $\mathrm{P} 450$, drug metabolism, propanoate metabolism, glyoxylate and dicarboxylate metabolism, tryptophan metabolism, leukocyte transendothelial migration, fatty acid elongation in the mitochondria, regulation of the actin cytoskeleton, fatty acid metabolism, pentose phosphate pathway, neurotrophin signaling pathway, fructose and mannose metabolism, cell cycle, and focal adhesion. The upregulated proteins in the 24-hour data set implicated eleven pathways, six of which were considered to be significant and are involved in antigen processing and presentation, tight junctions, MAPK pathway, endocytosis, adherens junctions, and ribosome. In the 28-day group (Figure 6), eight downregulated pathways 

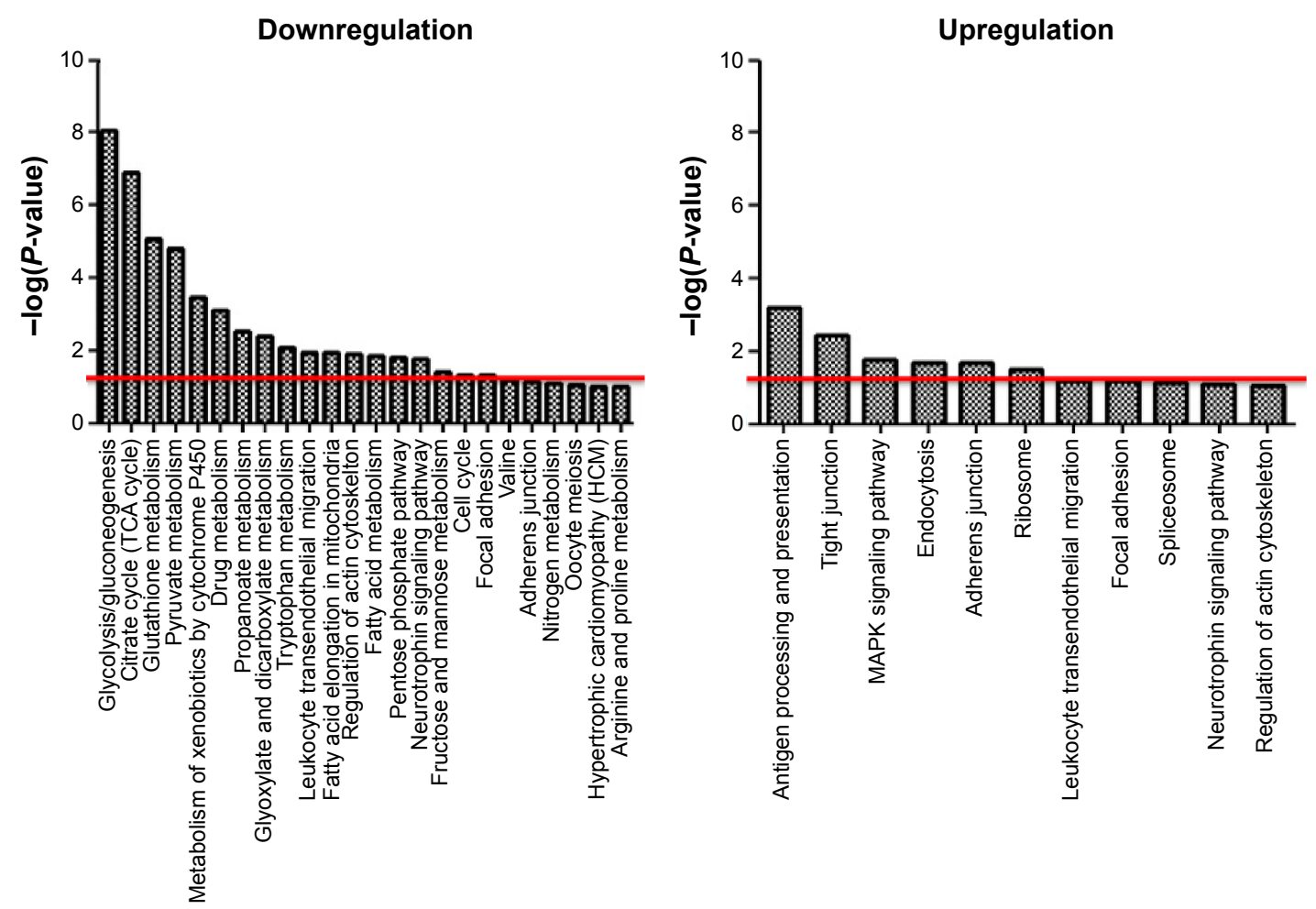

Figure 5 The pathways associated with the downregulation and upregulation of the protein responses 24 hours after exposure to $\mathrm{ZnONP}$.

Notes: The pathways were determined using DAVID analysis. An enhanced score (- $\log [P$-value $]) \geq 1.3$ threshold (red line) was considered to be significant. Abbreviations: ZnONP, zinc oxide nanoparticle; TCA, tricarboxylic acid; DAVID, Database for Annotation, Visualization and Integrated Discover.
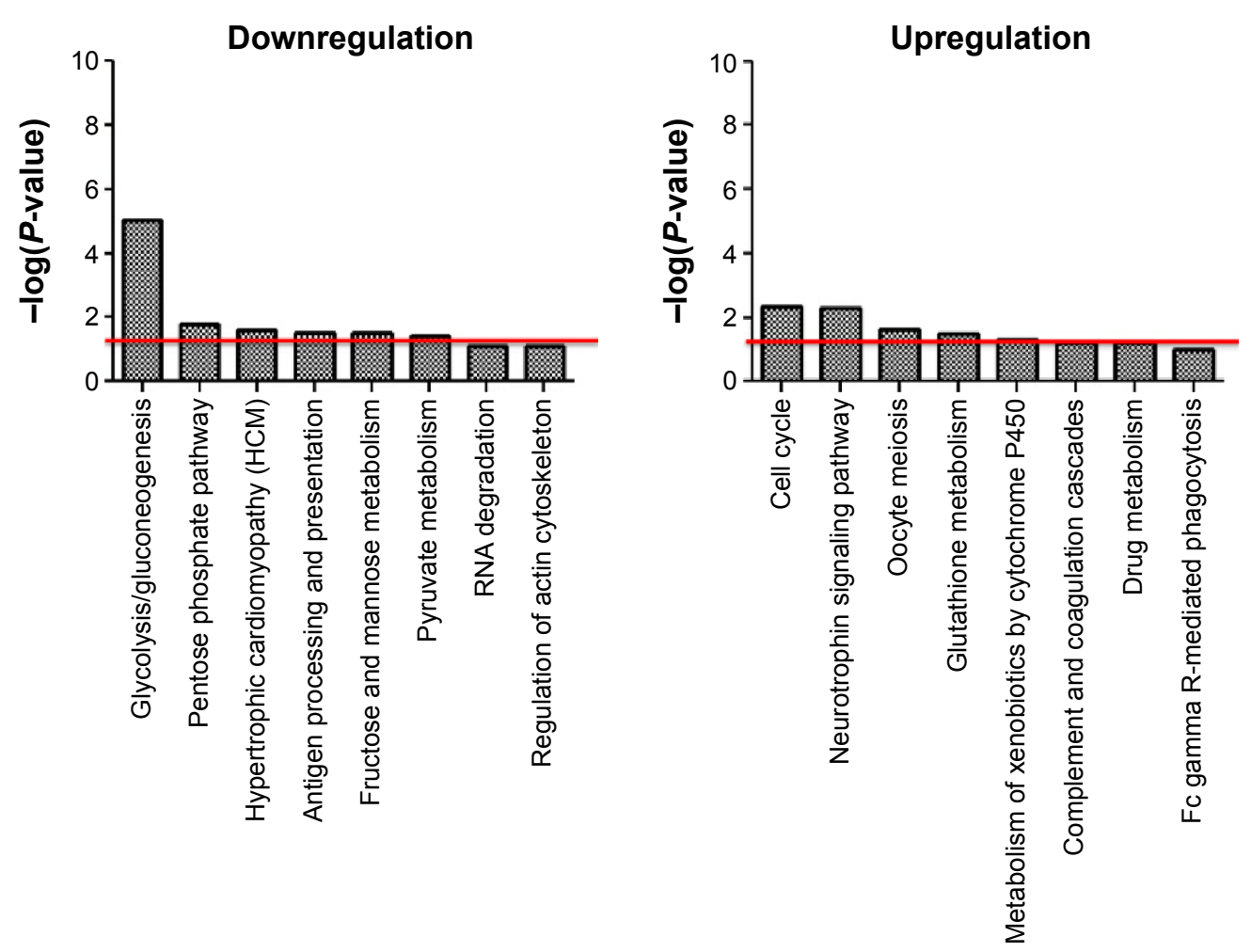

Figure 6 The pathways associated with the downregulation and upregulation of protein responses 28 days after exposure to $\mathrm{ZnONP}$.

Notes: The pathways were determined using DAVID analysis. An enhanced score (- $\log [P$-value]) $\geq 1.3$ threshold (red line) was considered to be significant. Abbreviations: ZnONP, zinc oxide nanoparticle; RNA, ribonucleic acid; DAVID, Database for Annotation, Visualization and Integrated Discover. 
were identified, with significance being observed for six. These included glycolysis/gluconeogenesis, pentose phosphate pathway, hypertrophic cardiomyopathy (HCM), antigen processing and presentation, fructose and mannose metabolism, and pyruvate metabolism. Regarding the upregulation in the 28-day group, cell cycle, neurotrophin signaling pathway, oocyte meiosis, glutathione metabolism, and metabolism of xenobiotics by cytochrome P450 were identified as significant. In a comparison between the significant pathways for the 24- and 28-day groups, we observed that the downregulated pathways for both the time frames considered included glycolysis/gluconeogenesis, pentose phosphate pathway, fructose and mannose metabolism, and pyruvate metabolism, whereas antigen processing and presentation which was upregulated in the 24-hour group became downregulated in the 28-day group. Furthermore, cell cycle, neurotrophin signaling pathway, glutathione metabolism, and metabolism of xenobiotics by cytochrome P450 which were downregulated in the 24-hour group were upregulated in the 28-day group.

\section{Protein network for 24 hours and 28 days follow-up of exposure}

Four proteins were identified to play important roles in the protein network in the 24-hour group, namely, (Figure 7) calpastatin (gene name: $C s$ ), cytochrome c somatic (gene name: $C y c s$ ), malate dehydrogenase (gene name: $M d h 2$ ), and annexin A1 (gene name: Anxal). There are eight proteins that play important roles in regulation of the protein network in the 28-day group, namely, (Figure 8) $\beta$-enolase (gene name: Eno3), calmodulin (gene name: Calm1), creatine kinase M-type (gene name: $\mathrm{Ckm}$ ), cytochrome c somatic (gene name: $C y c s$ ), vimentin (gene name: Vim), actin cytoplasmic 1 (gene name:
$A c t b$ ), $\beta$-actin-like protein 2 (gene name: $A c t b l 2$ ), and tubulin $\alpha-1$ A chain (gene name: Tubala).

\section{Discussion and conclusion}

Carcinogenic and genotoxic potentials related to ZnONP have been reported previously; however, the underlying mechanisms responsible for the responses due to exposure to $\mathrm{ZnONP}$ are still unclear. Altered metabolism is considered to be an important mechanism in regulation of the carcinogenicity and genotoxicity of ZnONPs, ${ }^{20,21}$ but the long-term effects of altered metabolism after $\mathrm{ZnONP}$ exposure remain unclear. Additionally, most investigations of $\mathrm{ZnONP}$ toxicity have been designed as acute or as repeat case-control experiments. Proteomic techniques have been applied to nanotoxicity investigations. ${ }^{22,23}$ These methods provide comprehensive information to understand and therefore prevent the potentially deleterious health effects due to exposure in occupational and environmental settings. ${ }^{3}$ The main findings observed in the present study are that $\mathrm{ZnONP}$ induced S100-A9 protein upregulation after 24 hours exposure; that metabolic processes and catalytic activity are the main biological processes and molecular functions, respectively, that are affected in the response to $\mathrm{ZnONP}$ exposure at the 24-hour and 28-day follow-up periods; and that downregulation of the glycolysis/gluconegeogenesis pathway is sustained during the period from 24 hours to 28 days after ZnONP exposure.

The physicochemical features of the $20 \mathrm{~nm}$ diameter engineered $\mathrm{ZnONP}$ were investigated. The SEM images showed that the ZnONPs were in the form of hexagonal columns and contained $\mathrm{Zn}$ and $\mathrm{O}$. Previous reports have suggested that the physicochemical properties of ZnONPs,

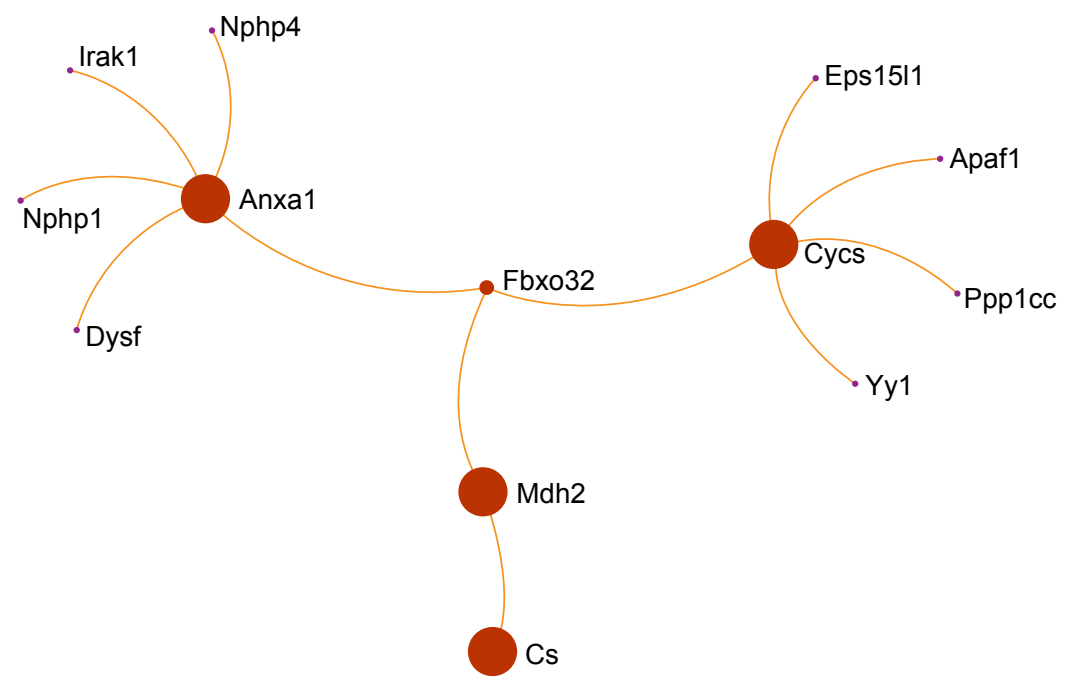

Figure 7 Protein network for 24 hour follow-up of exposure. 


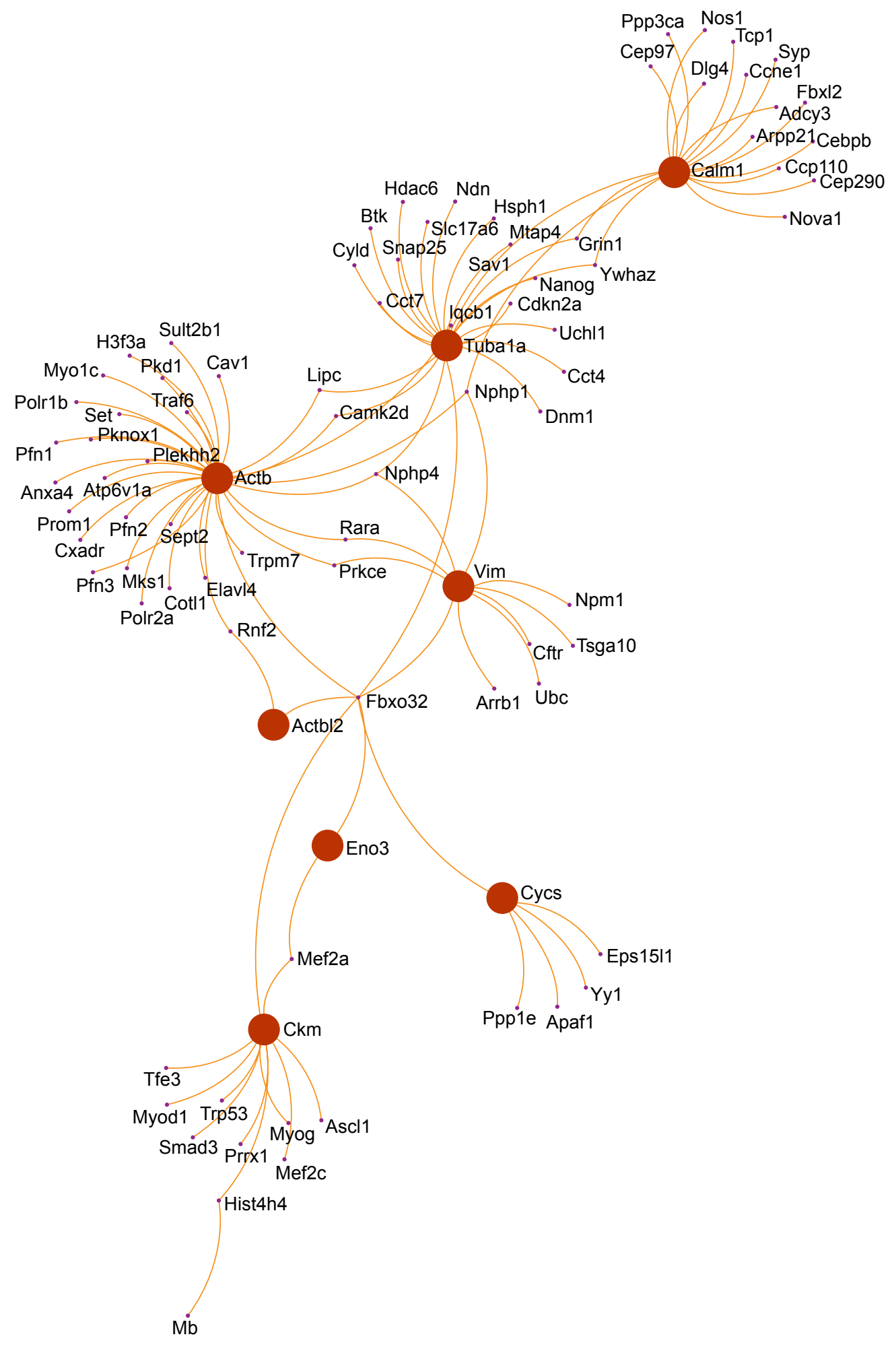

Figure 8 Protein network for 28-day follow-up of exposure.

including surface area, solubility, and $\mathrm{pH}$, play important roles in the regulation of their toxicity. ${ }^{24-26} \mathrm{We}$ have previously shown that a 14-day continuous exposure of rats to ZnONPs caused significant cardiopulmonary impairment observed after a 28-day follow-up period. ${ }^{9}$ However, the mechanisms underlying the acute and subacute effects of ZnONP exposure may be different. Also, a previous study showed that there was $0.14 \%$ of ${ }^{65} \mathrm{ZnONPs}$ remaining in the lungs after 28 days post-IT exposure. ${ }^{6}$ To determine the acute and subacute effects of the ZnONP on protein expression 
after a high-dose acute ZnONP exposure, $100 \mu \mathrm{g} / \mathrm{lung}$ ZnONPs were administered to the mice via IT instillation, and the effects were evaluated 24 hours and 28 days later. The doses applied in the present study were relevant for occupational exposure scenarios in humans. ${ }^{27}$ The mice were exposed to $100 \mu \mathrm{g} /$ lung ZnONPs (alveolar surface area $0.05 \mathrm{~m}^{2}$ ), which is equal to $200 \mathrm{mg} / \mathrm{lung} \mathrm{ZnONPs}$ in humans (alveolar surface area $102 \mathrm{~m}^{2}$ ). Considering the breathing frequency (30 times/min), lung deposition rate (30\%), a minute ventilation of $20 \mathrm{~L} / \mathrm{min}$, and the recommended $\mathrm{ZnO}$ standard of $5 \mathrm{mg} / \mathrm{m}^{3},{ }^{1}$ a human-equivalent dose would be achieved 13.6 days in a ZnONP-contaminated occupational environment.

In the lung samples, a total of 280 and 141 proteins were identified and quantified in the 24-hour and 28-day follow-up groups, respectively, using the iTRAQ-coupled LC-MS/MS approach. A total of 18 and 14 proteins displayed significant changes in the lung tissues in the 24-hour follow-up and 28-day follow-up groups, respectively. Using the iTRAQ-labeled proteomic approach, Juang et al ${ }^{28}$ identified 46 proteins in the bronchoalveolar lavage fluids (BALF) of rats that changed significantly after repeated exposure to ZnONPs. A transcriptional switch to an upregulation for neutrophil chemotactic genes was found and included genes such as S100A8, S100A9, and MMP9 after mice were exposed to welding fume. ${ }^{29}$ Consistent with our findings, S100-A9 protein was significantly upregulated 24 hours after the exposure to ZnONPs. S100-A9 protein is secreted from neutrophils, macrophages, and epithelial cells. These cell types are involved in various inflammatory diseases ${ }^{30}$ and in the development of lung cancer. ${ }^{31}$ Additionally, fatty acid-binding protein was significantly downregulated 24 hours after exposure. Fatty acid-binding protein is a known intracellular lipid chaperone. These proteins are a group of molecules that coordinate lipid responses in cells and are associated with metabolic and inflammatory pathways. ${ }^{32,33}$ With regard to the 28 days time point after exposure, we observed that tubulin $\alpha-1 \mathrm{~A}$ chain was significantly upregulated in the lungs, whereas carbonic anhydrase-3 was significantly downregulated. Carbonic anhydrase contains a zinc ion in its active site and serves to interconvert carbon dioxide and bicarbonate to maintain the circulating acid-base balance. Additionally, carbonic anhydrase assists in the transportation of carbon dioxide out of tissues. Dysfunctional carbonic anhydrase has been reported to cause metabolic dysfunction. ${ }^{34}$

The effects on these highly regulated proteins suggest that metabolic and inflammatory mechanisms may be important in the responses to ZnONPs at the 24-hour and 28-day time points after exposure. Importantly, metabolic processes and catalytic activity were the main biological processes and molecular functions implicated by both the downregulated and upregulated proteins at the 24-hour and 28-day time points after exposure to ZnONPs. These two main biological and molecular responses have also been identified after exposure of human epithelial A549 cells to ZnONPs. ${ }^{26}$

The iTRAQ proteomic results obtained from this study provide biological and molecular information on commonly used $\mathrm{ZnONP}$ that may be of relevance to toxicity or pathways of metabolism induced by ZnONPs, which may lead to inflammatory responses. We further showed that glycolysis/gluconeogenesis, pentose phosphate pathway, fructose and mannose metabolism, and pyruvate metabolism were downregulated at the 24-hour and 28-day time points after exposure. Furthermore, cell cycle, neurotrophin signaling pathway, glutathione metabolism, and metabolism of xenobiotics by cytochrome $\mathrm{P} 450$ which were downregulated at 24 hours became upregulated at 28 days after exposure. First, the downregulated metabolic pathways included glycolysis/ gluconeogenesis, pentose phosphate pathway, fructose and mannose metabolism, and pyruvate metabolism. Glycolysis, for example, converts glucose into pyruvate. The free energy released in this process is used to generate ATP (adenosine triphosphate) and NADH (reduced nicotinamide adenine dinucleotide) ${ }^{35}$ Similarly, alteration in glycolysis have been observed following exposure to copper oxide NPs ${ }^{36}$ and titanium dioxide NPs. ${ }^{23}$ Yang et al (2010) observed that silicon dioxide NPs caused dysfunctional energy metabolism in $\mathrm{HaCaT}$ cells, and they suggested that glycolysis could be a potential pathway in response to NPs. ${ }^{37}$ Notably, changes in the major pathways involved in detoxification after ZnONP exposure were also observed. These pathways included cell cycle, neurotrophin signaling pathway, glutathione metabolism, and cytochrome P450-mediated metabolism of xenobiotics. Regulation of the cell cycle is an important process for the prevention of cytotoxicity/genotoxicity induced by toxicants. The checkpoints in the cell cycle include one in the Sub- $G_{1}$ phase, which is activated in the late stage in the apoptotic cascade due to endonucleases breaking the connection between nucleosomes. ${ }^{38} \mathrm{ZnONP}$-driven oxidative stress causes alterations in the $\mathrm{p} 53$, survivin, bax/bcl-2, and caspase pathways in A549 cells, ${ }^{39}$ which lead to cell death. The neurotrophins, a family of closely related proteins, were initially identified as survival factors for sensory and sympathetic neurons. ${ }^{40}$ Neurotrophins are also related to survival, development, and functioning of neurons in both the peripheral and the central nervous systems. ${ }^{40}$ Disruption of cellular 
zinc homeostasis and downregulation of the GAP-43 protein have been reported in response to $\mathrm{ZnONP}$ exposure in rat pheochromocytoma PC12 cells. ${ }^{41}$ This effect might be associated with the balance in the biological levels of zinc ions. Glutathione metabolism and metabolism of xenobiotics by cytochrome P450 are important pathways in regulation of oxidative stress in cells. An overload of free radicals could result in a deficiency in glutathione. Glutathione is a signal for the initiation of apoptosis ${ }^{42}$ and, in addition to reactive oxygen species, could serve as a critical apoptotic mediator; particularly when they occur in the mitochondria, these changes would cause irreversible apoptosis.

In conclusion, we have shown that the S100-A9 protein was upregulated 24 hours after exposure of ZnONPs; metabolic processes and catalytic activity are the major response to $\mathrm{ZnONP}$ in 24 hours and 28 days after exposure; and the major mechanisms underlying these changes are those involved in the glycolysis/gluconegeogenesis pathway and regulation of the cell cycle. However, the resultant inflammation was not determined in the present study, and future work is required. We also provide a comprehensive understanding of the potential timedependent effects of exposure to ZnONPs. Our findings have significant implications for discovering the biological and molecular responses responsible for the effects of $\mathrm{ZnONPs}$, and continued work is required to more thoroughly elucidate the relationships between these processes and the epidemiological observations that have been observed.

\section{Acknowledgments}

The authors thank Miss Yi-Syuan Lin, His-En Hua, and Yen-Ling Shen for technical assistance during this project. The authors are grateful of technical support from Animal Molecular Imaging Core Facility at Taiwan National Health Research Institutes (NM-103-PP-04) and for intelligent input from Mr Wei-Neng Liao (NM-103-PP-07). This study was funded by the Institute of Occupational Safety and Health, Taiwan Council of Labor Affairs (IOSH102-M321 and IOSH103-M312).

\section{Author contributions}

All authors have contributed substantially to the concept, design, drafting the article, and critical revision of the paper for important intellectual content. All authors read and approved the final version of the paper for publication.

\section{Disclosure}

The authors report no conflicts of interest in this work.

\section{References}

1. Fine JM, Gordon T, Chen LC, et al. Metal fume fever: characterization of clinical and plasma IL-6 responses in controlled human exposures to zinc oxide fume at and below the threshold limit value. $J$ Occup Environ Med. 1997;39:722-726.

2. Grommes J, Soehnlein O. Contribution of neutrophils to acute lung injury. Mol Med. 2011;17:293-307.

3. Kwon JY, Koedrith P, Seo YR. Current investigations into the genotoxicity of zinc oxide and silica nanoparticles in mammalian models in vitro and in vivo: carcinogenic/genotoxic potential, relevant mechanisms and biomarkers, artifacts, and limitations. Int J Nanomedicine. 2014;9:271-286.

4. Ahsan SA, Lackovic M, Katner A, et al. Metal fume fever: a review of the literature and cases reported to the Louisiana Poison Control Center. J La State Med Soc. 2009;161:348-351.

5. Benninghoff A. Toxicoproteomics - the next step in the evolution of environmental biomarkers. Toxicol Sci. 2007;95:1-4.

6. Chen S, Guttridge DC, You Z, et al. Wnt-1 signaling inhibits apoptosis by activating beta-catenin/T cell factor-mediated transcription. $J$ Cell Biol. 2001;152:87-96.

7. Yang K, Hitomi M, Stacey DW. Variations in cyclin D1 levels through the cell cycle determine the proliferative fate of a cell. Cell Div. 2006;1:32.

8. Imoto M, Tanabe K, Simizu S, et al. Inhibition of cyclin D1 expression and induction of apoptosis by inostamycin in small cell lung carcinoma cells. Jpn J Cancer Res. 1998;89:315-322.

9. Chuang HC, Juan HT, Chang CN, et al. Cardiopulmonary toxicity of pulmonary exposure to occupationally relevant zinc oxide nanoparticles. Nanotoxicology. 2014;8:593-604.

10. Kim YH, Fazlollahi F, Kennedy IM, et al. Alveolar epithelial cell injury due to zinc oxide nanoparticle exposure. Am J Respir Crit Care Med. 2010;182:1398-1409.

11. Li N, Wang M, Bramble LA, et al. The adjuvant effect of ambient particulate matter is closely reflected by the particulate oxidant potential. Environ Health Perspect. 2009;117:1116-1123.

12. Tsai SS, Tiao MM, Kuo HW, et al. Association of bladder cancer with residential exposure to petrochemical air pollutant emissions in Taiwan. J Toxicol Environ Health A. 2009;72:53-59.

13. Huang YC, Li Z, Harder SD, et al. Apoptotic and inflammatory effects induced by different particles in human alveolar macrophages. Inhal Toxicol. 2004;16:863-878.

14. Weng HH, Tsai SS, Chiu HF, et al. Association of childhood leukemia with residential exposure to petrochemical air pollution in taiwan. Inhal Toxicol. 2008;20:31-36.

15. Chen T-T, Chuang K-J, Chiang L-L, et al. Characterization of the interactions between protein and carbon black. J Hazard Mater. 2014;264: $127-135$.

16. Huang da W, Sherman BT, Tan Q, et al. DAVID Bioinformatics Resources: expanded annotation database and novel algorithms to better extract biology from large gene lists. Nucleic Acids Res. 2007;35: W169-W175.

17. Srivastava R, Ray S, Vaibhav V, et al. Serum profiling of leptospirosis patients to investigate proteomic alterations. J Proteomics. 2012;76: $56-68$.

18. Huang da W, Sherman BT, Lempicki RA. Systematic and integrative analysis of large gene lists using DAVID bioinformatics resources. Nat Protoc. 2009;4:44-57.

19. Xia J, Benner MJ, Hancock RE. NetworkAnalyst - integrative approaches for protein-protein interaction network analysis and visual exploration. Nucleic Acids Res. 2014;42:W167-W174.

20. Yang CY, Wang JD, Chan CC, et al. Respiratory symptoms of primary school children living in a petrochemical polluted area in Taiwan. Pediatr Pulmonol. 1998;25:299-303.

21. Yang CY, Cheng BH, Hsu TY, et al. Association between petrochemical air pollution and adverse pregnancy outcomes in Taiwan. Arch Environ Health. 2002;57:461-465.

22. Su CL, Chen TT, Chang CC, et al. Comparative proteomics of inhaled silver nanoparticles in healthy and allergen provoked mice. Int $J \mathrm{Nano}-$ medicine. 2013;8:2783-2799. 
23. Ahamed M, Alhadlaq HA. Nickel nanoparticle-induced dose-dependent cyto-genotoxicity in human breast carcinoma MCF-7 cells. Onco Targets Ther. 2014;7:269-280.

24. Buerki-Thurnherr T, Xiao L, Diener L, et al. In vitro mechanistic study towards a better understanding of $\mathrm{ZnO}$ nanoparticle toxicity. Nanotoxicology. 2013;7(4):402-416.

25. Ho M, Wu KY, Chein HM, et al. Pulmonary toxicity of inhaled nanoscale and fine zinc oxide particles: mass and surface area as an exposure metric. Inhal Toxicol. 2011;23:947-956.

26. Pan CH, Liu WT, Bien MY, et al. Effects of size and surface of zinc oxide and aluminum-doped zinc oxide nanoparticles on cell viability inferred by proteomic analyses. Int J Nanomedicine. 2014;9:3631-3643.

27. Rovira E, Cuadras A, Aguilar X, et al. Asthma, respiratory symptoms and lung function in children living near a petrochemical site. Environ Res. 2014;133:156-163.

28. Juang YM, Lai BH, Chien HJ, et al. Changes in protein expression in rat bronchoalveolar lavage fluid after exposure to zinc oxide nanoparticles: an iTRAQ proteomic approach. Rapid Commun Mass Spectrom. 2014;28:974-980.

29. Zeidler-Erdely PC, Kashon ML, Li S, et al. Response of the mouse lung transcriptome to welding fume: effects of stainless and mild steel fumes on lung gene expression in $\mathrm{A} / \mathrm{J}$ and $\mathrm{C} 57 \mathrm{BL} / 6 \mathrm{~J}$ mice. Respir Res. 2010;11:70

30. Chio CP, Yuan TH, Shie RH, et al. Assessing vanadium and arsenic exposure of people living near a petrochemical complex with two-stage dispersion models. J Hazard Mater. 2014;271:98-107.

31. Yang JJ, Liu CC, Chen WH, et al. Assessing the altitude effect on distributions of volatile organic compounds from different sources by principal component analysis. Env Sci Process Impact. 2013;15:972-985.

32. Yen $\mathrm{CH}$, Horng JJ. Volatile organic compounds (VOCs) emission characteristics and control strategies for a petrochemical industrial area in middle Taiwan. J Environ Sci Health A Tox Hazard Subst Environ Eng. 2009;44:1424-1429.
33. Liao MF, Liao MN, Lin SN, et al. Prevalence of allergic diseases of schoolchildren in central taiwan. From ISAAC surveys 5 years apart. J Asthma. 2009;46:541-545.

34. Weng HH, Tsai SS, Chiu HF, et al. Association of childhood leukemia with residential exposure to petrochemical air pollution in taiwan. Inhal Toxicol. 2008;20:31-36.

35. Liu CC, Chen $\mathrm{CC}, \mathrm{Wu} \mathrm{TN}$, et al. Association of brain cancer with residential exposure to petrochemical air pollution in Taiwan. J Toxicol Environ Health A. 2008;71:310-314.

36. Yu CL, Wang SF, Pan PC, et al. Residential exposure to petrochemicals and the risk of leukemia: using geographic information system tools to estimate individual-level residential exposure. Am J Epidemiol. 2006;164:200-207.

37. Yang $\mathrm{X}$, Liu $\mathrm{J}, \mathrm{He} \mathrm{H}$, et al. $\mathrm{SiO}_{2}$ nanoparticles induce cytotoxicity and protein expression alteration in HaCaT cells. Part Fibre Toxicol. 2010;7:1

38. Chuang HC, Jones T, Chen TT, et al. Cytotoxic effects of incense particles in relation to oxidative stress, the cell cycle and F-actin assembly. Toxicol Lett. 2013;220:229-237.

39. Manke A, Wang L, Rojanasakul Y. Mechanisms of nanoparticle-induced oxidative stress and toxicity. Biomed Res Int. 2013;2013:942916.

40. Madl AK, Plummer LE, Carosino C, et al. Nanoparticles, lung injury, and the role of oxidant stress. Annu Rev Physiol. 2014;76:447-465.

41. Shimada A, Kawamura N, Okajima M, et al. Translocation pathway of the intratracheally instilled ultrafine particles from the lung into the blood circulation in the mouse. Toxicol Pathol. 2006;34:949-957.

42. Langer C, Jurgensmeier JM, Bauer G. Reactive oxygen species act at both TGF-beta-dependent and -independent steps during induction of apoptosis of transformed cells by normal cells. Exp Cell Res. 1996;222:117-124.
International Journal of Nanomedicine

\section{Publish your work in this journal}

The International Journal of Nanomedicine is an international, peerreviewed journal focusing on the application of nanotechnology in diagnostics, therapeutics, and drug delivery systems throughout the biomedical field. This journal is indexed on PubMed Central, MedLine, CAS, SciSearch ${ }^{\circledR}$, Current Contents ${ }^{\circledR} /$ Clinical Medicine,

\section{Dovepress}

Journal Citation Reports/Science Edition, EMBase, Scopus and the Elsevier Bibliographic databases. The manuscript management system is completely online and includes a very quick and fair peer-review system, which is all easy to use. Visit http://www.dovepress.com/ testimonials.php to read real quotes from published authors. 